\title{
CALL FOR PAPERS 2022
}

The editors of the Bolivian Studies Journal invite the academic community to submit original research papers for volume 28, 2022. The journal welcomes original manuscripts that have not been submitted simultaneously to other publications, whether print or electronic.

\section{Deadline for submissions: June 30, 2022}

Manuscripts should be sent electronically directly to the website of the journal according to the editorial standards available at: http://bsj.pitt.edu/ojs/index.php/bsj/about/submissions\#onlineSubmis sions

For additional information on submissions and other matters related to the journal, visit the website (http://bsj.pitt.edu) or send a message to bsj@mail.pitt.edu 


\section{CONVOCATORIA 2022}

Se invita a la comunidad académica a someter manuscritos inéditos de investigación para el volumen 28, 2022. La Revista de Estudios Bolivianos requiere que estos trabajos no sean presentados simultáneamente a otras publicaciones, sean éstas impresas o electrónicas.

\section{Recepción de manuscritos hasta el 30 de junio de 2022}

Los manuscritos deberán ser enviados por vía electrónica directamente a la página web de la revista de acuerdo a las normas editoriales disponibles en:

http://bsj.pitt.edu/ojs/index.php/bsj/about/submissions\#onlineSubmis sions

Para información adicional sobre envío de manuscritos y otros asuntos relacionados con la revista, visitar la página web (http://bsj.pitt.edu) o dirigir su mensaje a: bsj@mail.pitt.edu 